\title{
PREVIOUS STRESS AND ACUTE PSYCHOLOGICAL DEFENCE AS PREDICTORS OF PERINATAL GRIEF-AN EXPLORATORY STUDY
}

\author{
J. A. M. Hunfeld, ${ }^{1.2 *}$ J. W. Wladimiroff, ${ }^{1}$ F. Verhage ${ }^{2}$ and J. Passchier ${ }^{2}$ \\ ${ }^{1}$ Department of Obstetrics and Gynaecology, University Hospital Dijkzigt, Rotterdam, The Netherlands \\ and ${ }^{2}$ Department of Medical Psychology and Psychotherapy, Erasmus University, Rotterdam, \\ The Netherlands
}

\begin{abstract}
We evaluated whether the emotional reactions of women at 2-6 weeks after the prenatal diagnosis of a lethal anomaly and at 3 months after perinatal loss might be predicted by previous stress and acute psychological defence reactions to the diagnosis. Previous stress was defined objectively as a history of major life event(s) and having received professional mental health treatment in the past, and subjectively as the disposition for feelings of inadequacy and anxiety. Forty-one women were interviewed and completed measures on their history of major life events, whether they had received professional mental health treatment in the past, inadequacy, acute psychological defence reactions and perinatal grief. Regression analyses showed that inadequacy was the most strongly positive predictor of perinatal grief shortly after receiving the unfavourable diagnosis and three months after perinatal loss. In addition to inadequacy, having received professional mental health treatment in the past led to significantly more intense grief, but only shortly after receiving the unfavourable diagnosis. Previous life events intensified grief three months after perinatal death. The grieving process was significantly moderated by the defence of 'principalization' while it was significantly intensified by 'turning aggression against oneself', but only shortly after receiving the unfavourable diagnosis.

These effects were not contaminated by relationships with pregnancy-related variables. Our findings imply that psychological support for women with perinatal loss should particularly be offered to those who have been identified as generally anxious, who have reported previous major life events and have received professional mental health treatment in the past.
\end{abstract}

Key words - perinatal grief, acute psychological defence, previous stress

\section{INTRODUCTION}

Our previous study showed that the emotional reactions of 41 women to the stress of infant loss owing to severe fetal malformations, were still strong three months after delivery and the subsequent death of the infant [1]. In addition, we found more grief in women whose gestational age lay between 24 or 34 weeks (vs beyond 34 weeks), in women who had viewed the dead infant (vs not viewed the dead infant) and in women who had not experienced any difficulty with conceiving (vs difficulty with conceiving).

Apart from these factors which are directly related to pregnancy, previous stressful life events of the mother might lead to disordered mourning. $\dagger$

*Author for correspondence at Department of Medical Psychology and Psychotherapy, Erasmus University, Rotterdam, Postbus 1738, 3000 DR Rotterdam, The Netherlands.

†The term 'mourning' refers to a complex interplay of all the psychological processes that are triggered by the loss. This includes biological reactions, behavioural reactions, and cognitive and defensive operations related to the loss. The term 'grief' refers to all the painful affects associated with the loss of an infant, such as sadness, anger, guilt, shame and anxiety [2].
Although perinatal loss was at first seen as a "non event' [3], previous perinatal loss is now considered to be an important factor in a troublesome grieving process [4-7]. Further, experiences of incest can lay the foundation for complicated mourning in adult life [8]. It might be questioned whether previous life events in general also lead to more intense grieving.

In addition, research findings generally suggest that stress-related personality characteristics, such as an obsessive personality or insecure, anxious-attached and fearful individuals, sometimes labelled as neurotic $[9,32]$, may be at higher risk for troublesome grieving than individuals without these features [4,9-11]; see also recent reviews on bereavement [7, 12-14]. However, there is little systematic knowledge on the relationship between inadequacy and coping with perinatal loss. Many studies have assessed this relationship without using any specific standardized measure or statistical test $[4,7,12-14]$. Owing to the fact that previous use of professional mental health treatment generally indicates that the psychological burden of life outweighs the psychological carrying capacity of an individual [15], it can be hypothesized that women who have received professional mental health treatment at some time in the 
past, might suffer more distress after later perinatal loss than women who have not. Black [16] had not found a significant association between previous use of professional mental health treatment and distress. However, the association was determined only at a second measurement 6 months after pregnancy loss and by means of the POMS which is a general mood scale. It might be questioned whether this relationship is also absent if assessed shortly after hearing the unfavourable diagnosis and by means of a specific perinatal grief scale.

Being informed of an unfavourable diagnosis is a heavy blow to the mother, so she will mobilize several psychological defences against the situation to protect herself from being overwhelmed by painful emotions, such as fear, guilt and anger [17]. Particularly in the beginning, defences against painful emotions are considered to be a useful part of coping, but they might also lead to delayed or disordered mourning, for example by continual suppression of the unpleasant feelings and thoughts related to perinatal loss [18]. So far, no studies are available on perinatal grief in relation to psychological defences. Therefore the present study addressed two questions:

(1) What is the relationship between previous stressful life events, inadequacy and having received professional mental health treatment in the past on the one hand and perinatal stress and grief on the other?

(2) What is the relationship between acute psychological defences against the unfavourable ultrasound diagnosis, and perinatal stress and grief?

\section{METHOD}

\section{Patients}

We approached 55 women who were referred to the division of prenatal diagnosis in the period between January 1990 and August 1991 for an anomaly scan. Inclusion criteria for the study were: (i) a pregnancy of 24 weeks or more; and (ii) the presence of a fetal malformation which was not compatible with extrauterine life or would at least result in severe mental and/or physical handicap(s). Women with a previously known risk for congenital fetal structural malformations and women with insufficient knowledge of the Dutch language were excluded from the study. A total of 46 pregnant women consented to take part in the study. The remaining nine women did not participate in the study because they did not wish to be confronted with the traumatic event or the loss $(n=8)$ or because the woman and her partner were both deaf $(n=1)$.

\section{Procedure}

A medical psychologist (J.H.) paid a home visit to carry out an audiotaped interview 2-6 weeks (first measurement) after the women had been informed of the presence of a severe fetal malformation. To measure long-term emotional reactions, previous life events, inadequacy, whether the woman had received professional mental health treatment in the past and psychological defences, a second interview took place three months following the birth of the infant (second measurement). We chose the 3-month time interval between the first and second measurements because we expected that the women would be more able to provide biographical information by that time.

At the first and second measurements, questionnaires were left behind for completion. The women were instructed to contact the researcher (J.H.) if they had any problems regarding completion. The women received a reminder by telephone if the questionnaire had not been returned two weeks later.

\section{Measurement}

The interviews were semi-structured and included both open-ended items and closed-ended questions, which covered the history of life events and whether the woman had received professional mental health treatment in the past. The prevalence of major life events was determined by means of a semi-structured interview instead of a standard questionnaire, because the former method leads to better recall of the life events [19].

Grief reactions were measured with two questionnaires: the Perinatal Grief Scale and the Perinatal Event Scale. The perinatal Grief Scale is a translation of Toedter's Perinatal Grief Scale [20]. The scale consists of three subscales: active grief, difficulty with coping and despair. The total PGS score is a summation of each subscale score. The reliability and validity of the scale are good for the American and Dutch versions $[21,22]$. The Perinatal Event Scale, which was developed to measure stress after a traumatic event, is an elaboration of the Impact of Event Scale [23]. We anchored the items to the traumatic event of the (impending) perinatal loss, as was recommended by Horowitz [23] and labelled the outcome as perinatal stress. The scale consists of 15 items clustered around two subscales: intrusion and avoidance. The total PEL score consists of the summation of the subscale scores. The reliability and validity of the original scale are satisfactory.

Acute psychological defences were measured with an adapted version of the Defense Mechanism Inventory (DMI) [24] which registers reactions to threat or conflict. This was followed by four questions about the women's actual reactions, reactions in fantasy and reactions regarding thoughts and feelings upon hearing the unfavourable diagnosis (see Appendix). From the reactions which reflected the five major defences, the intensity of usage against the traumatic event was ranked: (i) turning aggression against a real or presumed external frustrating object* (TAO);

\footnotetext{
*The term 'object' comprises anything from human beings to things in the outside world through which gratification can be achieved.
} 
(ii) projection, which means attributing negative intent or characteristics to an external object, without unequivocal evidence (PRO); (iii) principalization (PRN) which refers to a mental process in which a person obscures, reinterprets or generalizes the affective meaning of a perceived threat. This class of defences deals with conflict through invoking a general principle that 'splits off' affect from content and represses the former. Defences such as intellectualization, isolation and rationalization fall into this category [26]; (iv) turning aggression against oneself (TAS) expressed in excessive self-criticism or self-reproach; (v) reversal (REV) which means a positive or neutral reaction to a traumatic event, while more negative reactions are expected. The defences TAO and PRO can be considered to measure hostile impulses directed towards other persons, while the defences PRN, TAS and REV can be considered to measure repression of the affect and self-directed aggression. The reliability and validity of the Dutch version are satisfactory for women [27]. In this study, we used instead of the general threat or conflict situations of the DMI the event of hearing the ultrasound diagnosis of a severe or lethal fetal anomaly, in order to increase the clinical validity [25].

Inadequacy was measured with the scales of social inadequacy (SI) and personal inadequacy (PI) which are subscales from the Dutch Personality Questionnaire. High scores on social inadequacy indicate incompetence in social contacts. These persons feel inhibited and shy. 'Personal inadequacy' items include questions about depressed mood, anxiety, feelings of insufficiency or low self-esteem. Both subscales can be considered to measure inadequacy or the disposition of the woman toward being anxious. The scales have good test-retest reliability and validity research has confirmed the intended content of the subscales [28].

\section{Data reduction}

A composite index for repression (REP) was calculated as the difference between (REV + PRN) and (TAO + PRO) since Juni reported this measure as being more reliable than the separate defence measures of the DMI [29].

The content of the audiotaped interview was first transcribed into a report from which the variables major life events and having received professional mental health treatment in the past were derived with two levels (present vs absent). Six major life events were registered; 5 of them had the highest rates of stressfulness in Paykel's life event scale [30]. These were: death of partner, offspring or parents; divorce and death of a good friend. Further, incest was added as a major life event.

\section{Data analysis}

First, the relationships between life events, inadequacy, having received professional mental health treatment in the past and acute defence measures on the one hand and the grief scores at the first and second measurements on the other, were assessed by Pearson's product moment correlations. Second, multiple regression analyses were carried out on the overall grief measures as dependent variables. Measures which proved to be significantly associated with both the independent variables and the grief scores were chosen as independent variables [31].

\section{RESULTS}

\section{Sample characteristics}

Maternal age in the sample $(n=46)$ ranged between 19-44 years (median: 30 yrs) and the gestational age varied between 24-38 weeks (median: $31 \mathrm{wks}$ ). Thirty-eight of the deliveries were induced, including four following intrauterine death. There were eight spontaneous deliveries. A total of five infants were alive during the study period, but were suffering from severe physical and/or mental handicap(s). A more detailed description of the sample is reported elsewhere [1].

At the time of the second measurement, five women withdrew from the study because they did not wish to be confronted with the loss. Therefore 41 women remained for further analysis.

Prevalence of previous stress, acute psychological defences, perinatal stress and grief

There was no significant reduction in perinatal stress and perinatal grief between the first and the second measurements (mean scores were 38.3 vs 33.5 and 83.2 vs 82.5 ). A more detailed description of the grief scores is reported elsewhere [1]. Twenty-six women $(63 \%)$ reported that they had experienced one or more major life events. Ten women $(24 \%)$ mentioned that they had received professional mental health treatment in the past. The mean score of social inadequacy was 11.7 (SD 8.1) and of personal inadequacy 15.5 (SD 10.8), which is within the normal range (12.3, SD 7.1 and 13.9, SD 8.3). The defence 'turning aggression against oneself' was most prominent in the reactions to hearing the diagnosis, followed by 'principalization', while the defences 'projection' and 'turning aggression against others' were least observed.

Relationship between previous stress, acute psychological defences and perinatal grief

Table 1 shows the relationship between major life events, inadequacy, having received professional mental health treatment in the past and defences on the one hand and perinatal stress and grief on the other. Inadequacy, particularly personal inadequacy, showed a significant and positive relationship with perinatal stress and grief shortly after hearing the unfavourable diagnosis and three months after the loss. The defence 'turning aggression against oneself' 


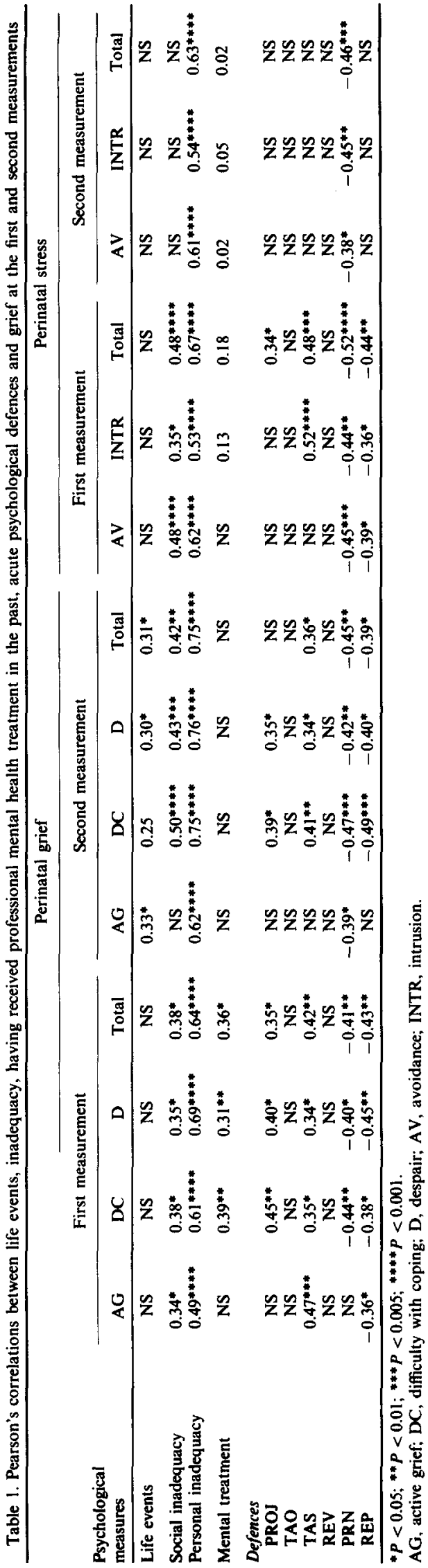

had a positive and significant association with perinatal grief at both measurements and with perinatal stress only shortly after the unfavourable diagnosis. At both measurements, significant negative correlations were observed between the defence principalization and perinatal stress and grief. Having received professional mental health treatment in the past was significantly and positively related to perinatal grief shortly after hearing the unfavourable diagnosis, while major life events showed a significant positive relationship with grief three months after perinatal loss.

Prediction of perinatal stress and grief using life events, inadequacy, having received professional mental health treatment in the past and acute psychological defences

The outcome of the multiple regression analyses is presented in Table 2. Personal inadequacy explained almost half of the variance in perinatal stress and grief scores shortly after hearing the unfavourable diagnosis. The women who displayed personal inadequacy showed significantly more grief than the women who did not. In addition, having received professional mental health treatment in the past made a unique and significant contribution to perinatal grief and accounted for $12 \%$ of the variance: the women who had received professional mental health treatment in the past showed more intense perinatal grief. In addition to personal inadequacy, the defences 'principalization' and 'turning aggression against oneself' made unique and significant contributions to the variance in perinatal stress: the women who reacted to the unfavourable diagnosis with 'principalization', showed less stress, while the women who 'turned aggression against themselves' showed more stress. Personal inadequacy also explained more than half of the variance in the perinatal grief scores three months after perinatal death. In addition, major life event(s) made a unique and significant contribution to perinatal grief: the women who had experienced major life events showed more intense grief than the women without a history of major life events. Personal inadequacy explained almost half of the variance in perinatal stress three months after perinatal loss, but none of the other variables made any significant contribution.

\section{DISCUSSION}

Inadequacy, previous life events and having received professional mental health treatment in the past led to more intense grief reactions. It has to be emphasized that our measure of inadequacy is not referring to psychopathology. These measures concern a general personality trait and indicate how a patient's position relates to that of the average population and whether it is in the low, middle, or high range of the distribution. Consequently, enhanced inadequacy scores in these women do not mean that 
they require psychotherapeutic treatment (although a subgroup of them might).

Of the above mentioned variables inadequacy, particularly personal inadequacy, was the strongest predictor of the variance in perinatal grief. In addition, a history of major life event(s) and having received professional mental health treatment in the past contributed to the variance in perinatal grief. Both variables were positively related to grief: having received professional mental health treatment in the past shortly after receiving the unfavourable diagnosis (first measurement) and previous life events three months after the perinatal loss (second measurement). In agreement with the finding of Black [16], the relationship between having received mental treatment in the past and grief had disappeared at the second measurement. The impact of having received mental treatment in the past might therefore be limited to crisis situations, such as receiving the unfavourable ultrasound diagnosis.

The finding that individuals with high scores on inadequacy showed more intense grief reactions is in agreement with research findings in other areas. These studies found more disordered grieving in neurotic women after an abortion and in neurotic soldiers after the Vietnam war $[9,32]$, both assessed by the Neuroticism subscale of the Eysenck Personality Questionnaire.

It was found that trauma often leads to the intrusive re-experience of earlier traumatic events [33, 34]; our findings indicated that this only took place after the first turmoil of the event had subsided. Rapee et al. [11] observed that anxious persons attributed a significantly more negative impact to life events than non-anxious persons. Unfortunately, our sample was too small to determine whether impact of life events, type of life event (loss by death, including perinatal loss, incest, divorce) or coming to terms with an event was influenced by the degree of inadequacy of the mother and made any difference to the grief reactions.

Only 'turning aggression against oneself' and 'prin- cipalization' among the acute psychological defences made a contribution to the variance in perinatal grief, in addition to personal inadequacy. 'Turning aggression against oneself' led to more intense grieving and 'principalization' to less intense grief reactions. As expected, this relationship was only found at the first measurement on our version of the Defense Mechanism Inventory which focussed on the psychological state after hearing the unfavourable diagnosis. The finding that the defences of 'turning aggression against oneself' and 'principalization' were related to respectively more and less intense grief is consistent with the viewpoint of a hierarchy of defences arranged according to their pathological significance. In this hierarchy, 'turning aggression against oneself' is regarded as an immature defence which is negatively related to mental health. 'Principalization' is considered to be a more mature defence and has no relationship with mental health according to Bond and Vaillant [25]. In this respect it is interesting that 'principalization' was not only significantly negatively related to 'intrusion' but also to 'avoidance' which supports Bond and Vaillant's consideration. It has to be noted that we measured the defences as a reflection of the psychological state in response to the diagnosis. It is salient that these state measures are predictive for the psychological reactions three months after the perinatal loss. Longitudinal research is necessary to reveal whether the defences reflect either a healthy coping process or delayed grieving.

Previous analyses on these women showed that several pregnancy-related variables, such as viewing the dead infant and no difficulty with conceiving, were positively and significantly related to the intensity of grief [1], therefore these variables may have confounded the relationships found in this study. However, entering these potential confounders into the analyses did not change the significant associations reported above. The outcome can therefore be considered to be independent of these pregnancyrelated variables.

A methodological weakness of this study is that the

Table 2. Life events, inadequacy, having received professional mental treatment in the past and acute psychological defences as predictors of perinatal stress and grief at the first and second measurements

\begin{tabular}{|c|c|c|c|c|}
\hline Psychological measures & $R^{2}$ & $R^{2}$ change & $F$ & $P$ \\
\hline \multicolumn{5}{|l|}{$\begin{array}{l}\text { First measurement } \\
\text { Perinatal grief }\end{array}$} \\
\hline Inadequacy & 0.37 & 0.37 & 18.67 & $<0.0001$ \\
\hline Mental health treatment & 0.60 & 0.12 & 7.01 & $<0.01$ \\
\hline \multicolumn{5}{|l|}{ Perinatal stress } \\
\hline Inadequacy & 0.35 & 0.35 & 17.90 & $<0.0002$ \\
\hline Principalization & 0.51 & 0.16 & 10.87 & $<0.002$ \\
\hline $\begin{array}{l}\text { Turning aggression } \\
\text { against oneself }\end{array}$ & 0.58 & 0.07 & 5.57 & $<0.02$ \\
\hline \multicolumn{5}{|l|}{$\begin{array}{l}\text { Second measurement } \\
\text { Perinatal grief }\end{array}$} \\
\hline Inadequacy & 0.54 & 0.54 & 36.99 & $<0.0001$ \\
\hline Life events & 0.61 & 0.07 & 5.54 & $<0.02$ \\
\hline \multicolumn{5}{|l|}{ Perinatal siress } \\
\hline Inadequacy & 0.39 & 0.39 & 24.72 & $<0.0001$ \\
\hline
\end{tabular}

$R^{2}$, explained variance.

$R^{2}$ change, increment in explained variance. 
inadequacy scores were collected in the same period as the grief scores, instead of before the traumatic event. The psychological state of the women might therefore have influenced the scores on the predictive measures. However, the mean scores on inadequacy largely fell within the average range of the norm scores for the general Dutch female population [28] and were not increased. This indicates that the scores of the women three months after delivery were also representative for anxiety before the diagnosis.

Another methodological aspect concerns our rather small number of patients. The relatively large number of variables tested implies that the findings have to be considered with caution. On the other hand, significant findings which are obtained in a small sample can indicate the presence of a large and probably clinical relevant effect.

From our findings, the association between inadequacy and grief was most outspoken. Other researchers have indicated that there is an association between inadequacy on the one hand and low social adjustment and not being able to provide structure and meaning to a traumatic event on the other [9, 32]. Further, it has been found that empathic listening to the women and labelling the emotions which they express concerening the perinatal loss, has a positive effect $[2,35]$. Our findings therefore imply that such an opportunity should be offered particularly to women who have been identified as generally anxious or having a disposition for feelings of inadequacy, who have reported (major) life events and have received professional mental health treatment in the past.

\section{CONCLUSION}

The emotional reactions to infant loss owing to severe fetal malformations are most strongly determined by inadequacy. In addition, having received professional mental health treatment in the past led to more intense grief reactions shortly after receiving the unfavourable diagnosis, while previous life events intensified the grief reactions three months after the loss. The grieving process was further intensified by the defence of 'turning aggression against oneself' and moderated by 'principalization' shortly after receiving the unfavourable diagnosis. Longitudinal research is necessary to reveal whether the defences reflect either a healthy coping process or delayed grieving.

Acknowledgements - This study is part of a larger research project on perinatal grief, which is supported by grants from the Dutch 'Ziekenfondsraad' (Ontwikkelings Geneeskunde) and the Dutch 'Nationaal Fonds voor de Geestelijke Volksgezondheid'.

\section{REFERENCES}

1. Hunfeld J. A. M., Wladimiroff J. W., Passchier J., Uniken Venema-Van Uden M. M. A. T., Frets P. G. and Verhage F. Emotional reactions in women in late pregnancy following the ultrasound diagnosis of a severe or lethal fetal anomaly. Prenatal Diagnosis 13, 603, 1993.

2. Zeanah C. H. Adaptation following perinatal loss: a critical review. J. Am. Acad. Child Adolescent Psychiat. 3, 467, 1989.

3. Lewis $\mathrm{E}$. The psychological consequences and strategies of management. Adv. Perinatal Med. 3, Monographs (Edited by Milunsky and Friedman), 1983.

4. Bowlby J. Attachment and Loss, III. Loss: Sadness and Depression, Reprint. Penguin Books, London, 1980.

5. Raphael B. The Anatomy of Bereavement. Hutchinson, London, 1984.

6. Nicol M. T., Thompkins J. R. et al. Maternal grieving response after perinatal death. Med. Austr. 144, 287, 1986.

7. Hall R. C. W., Beresford T. P. and Quinones J. E. Grief following spontaneous abortion. Psychiat. Clin. North Am. 10, 405, 1987.

8. Clark G. T., Cole G. and Enzle S. Complicated grief reactions in women who were sexually abused in childhood. J. Psychosoc. Oncol. 8, 87, 1990.

9. Friedman T. and Gath D. The psychiatric consequences of spontaneous abortion. Br. J. Psychiat. 155, 810, 1989.

10. Belitsky $R$. and Jacobs S. Bereavement, attachment theory and mental disorders. Psychiat. Ann. 16, 276, 1986.

11. Rapee R. M., Litwin E. M. and Barlow D. H. Impact of life events on subjects with panic disorder and on comparison subjects. Am. J. Psychiat. 147, 640, 1990.

12. Raphael B. and Middleton W. What is pathologic grief? Psychiatric Annals 20, 304, 1990.

13. Middleton W. and Raphael B. Bereavement. State of the art and state of the science. Psychiat. Clin. North Am. 10, 329, 1987.

14. Iles $\mathrm{S}$. The loss of early pregnancy. Bailliere's Clin. Obstetrics Gynaec. 3, 769, 1989.

15. Auslander G. K. Bereavement research in Israël: a critical review. Israel J. Psychiat. Related Sci. 24, 33, 1987.

16. Black R. B. A 1 and 6 month follow-up of prenatal diagnosis patients who lost pregnancies. Prenatal Diagnosis 9, 795, 1989.

17. Freud A. The ego and the mechanisms of defense. Writings of Anna Freud, 2. International Universities Press, New York, 1966.

18. Lindstrom T. C. Defence mechanisms and some notes on their relevance for the caring professions. Scand. $J$. Caring Sci. 3, 99, 1989.

19. Berden G. F. M. G. The development and application of a life event questionnaire and a life event interview. Dissertation, 1992.

20. Potvin L., Lasker J. N. and Toedter L. J. Measuring grief: a short version of the perinatal grief scale. J. Psychopath. Behav. Assessment 11, 29, 1989.

21. Toedter L. J., Lasker J. N. and Alhadeff M. A. The perinatal Grief Scale: development and initial validation. Am. J. Orthopsychiat. 58, 435, 1988.

22. Hunfeld J. A. M., Wladimiroff J. W., Passchier J., Uniken Venema-Van Uden M. M. A. T., Frets P. G. and Verhage $F$. Reliability and validity of a Dutch version of the Perinatal Grief Scale. Br. J. Med. Psychol. $66,295,1993$.

23. Horowitz M., Wilner B. A. and Alvarez W. Impact of Event Scale: a measure of subjective stress. Psychos. Med. 41, 209, 1979

24. Inilevich D. and Gleser G. C. Defenses in Psychotherapy: the Clinical Application of the Defense Mechanism Inventory. DMI Associates, Michigan, Ossowo, 1991.

25. Vaillant G. E. Ego Mechanisms of Defense. A Guide for Clinicians and Researchers. American Press, Washington, 1992. 
26. Gleser G. C. and Ihilevich D. An objective instrument for measuring defense mechanisms. J. Consulting Clin. Psychol. 33, 51, 1969.

27. Passchier J. and Verhage F. The Defense Mechanism Inventory: Preliminary findings on reliability and validity of the Dutch translation. Gedrag \& Gezondheid 14, $119,1986$.

28. Luteijn F., Starren J. and Van Dijk H. Guide to the Dutch Personatity Questionnaire. Swets \& Zeitlinger b.v., Lisse, 1985.

29. Juni S. The composite measure of the Defense Mechanism Inventory. J. Res. Personality 16, 193, 1982.

30. Paykel E. S., Prusoff B. A. and Uhlenhuth E. H. Scaling of life events. Arch. Gen. Psychiat. 25, 340, 1971.

31. Cohen J. and Cohen P. Applied Multiple Regression Correlation Analysis for the Behavioral Sciences. Lawrence Erlbaum Associates, Hillsdale, 1983.
32. Casella L., Motta R. W. Comparison of characteristics of Vietnam veterans with and without Posttraumatic Stress Disorder. Psychol. Rep. 67, 595, 1990.

33. Roy B., Peter P., Geraci M. and Uhde Th. W. Life events obtained via interview: the effect of time recall on data obtained in controls and patients with panic disorders. J. Affective Disorders 12, 57, 1987.

34. Theut S. K., Pedersen F. A., Zasiow M. J. and Rabinovich B. A. Pregnancy subsequent to perinatal loss: parental anxiety and depression. J. Am. Acad. Child Adolescent Psychiat. 27, 289, 1988.

35. Jorgensen $C_{\text {., Uddenberg }} \mathbf{N}$. and Ursing J. Diagnosis of fetal malformation in the 32nd week of gestation. A psychological challenge to the woman and the doctor. J. Psychos. Obstetrics Gynaec. 4, 73, 1985.

\section{APPENDIX}

The Adapted Version of the Defence Mechanism Inventory

QUESTION: How did you react at that time?

1. I burst into tears and wondered why it had to happen to me.

DEFENCE

TAS

2. I told the ultrasound doctor that what ever happened, I was pleased that it had been detected before the baby was born.

3. I didn't show much reaction at the hospital, because the doctors have to be able to do their jobs, too.

4. I was horrible to anyone who came near me at that time.

5. I asked to see the senior doctor, because I did not trust the result.

QUESTION: What would you have liked to have done impulsively in your fantasy?

6. I would like to have hit myself in the stomach.

REV

PRN

TAO

PROJ

7. I would like to have hit myself on the head.

TAO

TAS

8. I would like to have told the ultrasound doctor that I didn't trust him/her.

9. I would have liked to have run away, but I didn't dare.

PROJ

PRN

REV must be awful for him/her to have to tell somebody something like that.

QUESTION: What sort of thoughts did you have?

11. Why does something like this have to happen to me?

TAS

TAO

REV

PRN

14. Now it has happened to me, but it can happen to anyone.

PROJ

15. The baby is suffering from pain; I feel very sorry for it.

PROJ

TAS

REV

18. Thankful because I found out in advance through the ultrasound scan that there is something wrong.

19. Resigned, because that's just the way it is.

PRN

20. Angry about the way they told me the result.
TAO 